\title{
Stereotactic body radiotherapy for adrenal metastases from lung cancer
}

\author{
Michael Guiou • Nina A. Mayr • Edward Y. Kim • \\ Terence Williams • Simon S. Lo
}

Received: 13 February 2012 / Accepted: 26 April 2012 /Published online: 16 May 2012

(C) Springer-Verlag 2012

\begin{abstract}
Purpose The adrenal glands are common sites of metastatic disease in lung cancer and can be highly symptomatic. Current treatment approaches for adrenal oligometastases, including surgical resection and chemoembolization, are invasive and can be associated with considerable morbidity. More recently, stereotactic body radiotherapy (SBRT) has shown promising tumor control rates in primary lung cancer and oligometastases of various sites, but relatively less data exist on the efficacy of SBRT for adrenal metastases. The purpose of this study is to assess tumor regression pattern, local control, overall survival, pain relief, and treatment morbidity in patients treated with SBRT for adrenal metastases from lung cancer.

Methods and materials Eleven lesions were treated with SBRT in nine patients with lung cancer and followed with post-therapy clinical exams and computed tomography. Response Evaluation Criteria in Solid Tumors (RECIST)-based
\end{abstract}

M. Guiou $\cdot$ N. A. Mayr $\cdot$ T. Williams

Department of Radiation Oncology, Ohio State University,

Columbus, OH, USA

E. Y. Kim

Department of Radiation Oncology, University of Washington,

Seattle, WA, USA

S. S. Lo

Department of Radiation Oncology, University Hospitals Seidman Cancer Center, Case Western Reserve University,

Cleveland, OH, USA

N. A. Mayr ( $\square)$

Department of Radiation Oncology, Ohio State University, Arthur G. James Cancer Hospital and Solove Research Institute, 300 W 10th Ave, Rm. 078,

Columbus, OH 43210, USA

e-mail: Nina.Mayr@osumc.edu tumor response was assessed and volumetric tumor measurements were obtained by serial three-dimensional contouring. Symptomatic control, overall survival, and radiation therapyassociated side effects were assessed at follow-up visits. Mean post-therapy follow-up was 7.3 months.

Results The prescribed dose ranged from 20.0 to 37.5 Gy in

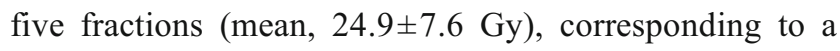
$\mathrm{BED}_{10}$ of 28.0 to 65.6 (mean, 41.6 \pm 11.6 ) Gy. Overall RECIST-based response rate was $67 \%$; 1-year and 2-year local control was $44 \%$; and 1-year and 2-year overall survival were $52 \%$ and $13 \%$, respectively. Volumetric response was much more rapid in small cell than in non-small cell carcinomas (slope, $-31.0 \%$ vs. $-5.9 \% /$ month, respectively, $p=0.06$ ). Patients with metachronous lesions had longer survival (1 year, 60\%; 2 year, 20\%) than patients with synchronous lesions ( 1 year, 38\%; 2 year, $0 \%$ ). No early or late grade $\leq 3$ adverse effects occurred.

Conclusion SBRT is a useful non-invasive treatment option for adrenal metastases from lung cancer, providing good local control with minimal morbidity. Small cell carcinoma lesions show rapid response that may require adaptive re-planning.

Keywords Adrenal metastases $\cdot$ Stereotactic body radiotherapy $\cdot$ Lung cancer $\cdot$ Palliative radiotherapy

\section{Introduction}

The adrenal glands are common sites of metastatic involvement in cancers arising in the skin (melanoma), lung, breast, and kidneys and other primary sites. These lesions can be bulky and can cause considerable pain, leading to decreased quality of life and significant narcotic usage [1-5]. Traditional treatment includes surgical resection, radiofrequency 
ablation, and chemoembolization [6-8]. However, these therapy approaches, particularly resection of tumor in the challenging to access retroperitoneal region, may not be an optimal option in patients, who are frequently debilitated from metastatic cancer and have a limited life expectancy [7].

Recently, stereotactic body radiotherapy (SBRT) approaches have provided a non-invasive alternative and broadened treatment options for patients with metastatic lesions in the lung, spine, liver, and other anatomic sites [9-18]. Excellent long-tem results in early stage primary lung cancer with local control rates equivalent to that of surgical resection have sparked interest in expanding the use of SBRT for metastatic lesions [19]. Similar high rates of local control have been recently reported for the use of SBRT in metastatic liver [17, 20,21] and spine lesions [11, 15, 22].

However, relatively less data exist on the efficacy of SBRT in the management of adrenal metastases from lung cancer. There is no prior experience on the regression rate and response dynamics of these frequently bulky tumors and the role of histology in their regression pattern, which are important considerations for the highly conformal stereotactic therapy delivery. The purpose of this study was to assess local control, overall survival, tumor volume regression pattern, symptomatic relief, and radiation-induced side effects in patients treated with SBRT for adrenal metastases from lung cancer.

\section{Methods and materials}

Patient population Following IRB approval, a retrospective review of nine patients with adrenal metastases from lung cancer was performed at our institution. Four women and five men were identified. Average age was 59.2 \pm 9.3 years. All patients had primary lung cancer (five small cell carcinoma (SCLC), four non-small cell carcinoma (NSCLC)) and had received previous therapy for their primary site of cancer. Seven of nine patients received definitive concurrent chemo/radiotherapy for their primary disease. One patient received palliative radiotherapy to the chest followed by chemotherapy and another had metastatic disease at diagnosis therefore received chemotherapy alone. At the time of treatment, systemic disease was stable in five patients and progressive in four. All of the patients were either poor surgical candidates or refused surgical intervention.

A total of ten lesions were treated. Eight patients had unilateral and one had bilateral adrenal metastases. Based on the study by Tanvetyanon et al. [23] that showed differences in survival dependent on disease-free interval (i.e., time from original diagnosis to diagnosis of adrenal lesions), the time between original diagnosis and diagnosis of the adrenal metastasis was scored as either metachronous or synchronous. Lesions were defined as synchronous if they

occurred $\leq 6$ months from time of diagnosis and metachronous of they were diagnosed $\geq 6$ months from time of diagnosis. Five lesions were metachronous and five were synchronous. Four patients presented with symptomatic, painful lesions requiring management with narcotics. Patient demographics are listed in Table 1.

Treatment Patients were immobilized using one of two methods. Five patients were simulated in the Elekta Body Frame (Elekta AB, Stockholm, Sweden) with abdominal compression to minimize respiratory motion. The remaining four patients were unable to tolerate compression due to body habitus, pain, or decreased respiratory function. These patients were simulated in a BlueBAG ${ }^{\mathrm{TM}}$ Vacuum Cushion (Elekta AB, Stockholm Sweden) with a knee sponge. All patients underwent 4D computed tomography (CT) for planning purposes. An internal target volume was generated that represented the maximal superior, inferior, and transverse movement of the lesion. This volume was then expanded to create the planning target volume (PTV). Dosimetric variables are presented in Table 2. Seven patients were treated using IMRT, and two were treated using three-dimensional (3D) conformal technique. Seven to ten, noncoplanar, $6 \mathrm{MV}$ photon beams were used. Prior to each fraction, patient setup was confirmed with conebeam CT. Eight of nine patients were treated every other day. One patient received a protracted course of treatment. Two fractions totaling 10 Gy were delivered prior to a break in treatment for a short course of palliative radiotherapy to a symptomatic neck mass. Two more fractions were delivered 1 month later after re-simulation and planning. On the final

Table 1 Patient characteristics

Of the nine patients, eight were available for post-treatment followup. One patient with non-small cell lung cancer (NSCLC) expired prior to first follow-up

\begin{tabular}{ll}
\hline Feature & Value \\
\hline Gender & \\
Male & 5 \\
Female & 4 \\
Age & \\
Mean & 59.2 \\
Range & $42-69$ \\
Primary site & \\
NSCLC & 4 \\
SCC & 5 \\
Metachronous lesions & 5 \\
Synchronous lesions & 5 \\
Systemic disease status & \\
Stable & 5 \\
Progressive & 4 \\
Painful lesion & \\
Yes & 4 \\
No & 5 \\
\hline
\end{tabular}


Table 2 Dosimetric variables

GTV gross tumor volume, PTV planning target volume, Gy Gray, $B E D_{10}$ Biological Equivalent Dose for an alpha/beta ratio of 10

fraction, the pre-treatment conebeam showed poor alignment therefore the treatment was re-planned, and the final 5-Gy fraction was delivered several weeks later. One patient received simultaneous treatment of $20 \mathrm{~Gy}$ in five fractions to the bilateral adrenal glands.

Post-therapy surveillance and follow-up Patients were followed approximately every 3 months with history and physical exam, pain and symptom assessment, and repeat imaging including CT of the abdomen and pelvis. Local disease response was determined by imaging response using Response Evaluation Criteria in Solid Tumors (RECIST)based criteria on follow-up scans [24]. In addition, 3D lesion volumes on the pre-therapy and serial post-therapy CT were compared over time.

\section{Data analysis}

For overall survival, death of any cause was scored, and loss to follow-up was censored. Overall survival was calculated as the time from completion of treatment to the date of death (as determined by clinician notes and Social Security Death Index). Local control was defined as the interval between completion of SBRT and increase in volume greater than $20 \%$ of the lesion on follow-up imaging. The rate of treatment response was determined by calculating the slope between the pre-SBRT and first follow-up values of the volumetric and 2D response curves. Survival and local control were calculated using the Kaplan-Meier analysis. Differences between patient groups were assessed with a two-tailed, Student's $t$ test.

\section{Results}

Treatment dose The prescription dose ranged from 20.0 to $37.5 \mathrm{~Gy}$ in five fractions (mean, $24.9 \pm 7.6 \mathrm{~Gy}$ ) prescribed to the perimeter of the adrenal PTV. This corresponded to a $\mathrm{BED}_{10}$ ranging from 28.0 to $65.6 \mathrm{~Gy}$ (mean, 41.6 $\pm 11.6 \mathrm{~Gy}$ ), utilizing standard linear quadratic modeling.

Objective response classification Mean follow-up was $7.3 \pm$ 7.8 months (range, 0-26 months). One patient died prior to first follow-up and was inevaluable. When response was assessed by RECIST criteria at 5 months after therapy completion for the remaining nine patients with ten lesions, there were six partial responses (PR), three patients with stable disease (SD), and none with progressive disease (PD), resulting in an objective response rate $(\mathrm{CR}+\mathrm{PR})$ of $67 \%$.

However, with assessment by volumetric measurement, response classification at 5 months was CR (0), PR (7), SD (1), and PD (1) for an objective response rate (CR+PR) of $78 \%$.

Among the three lesions (all NSCLC) classified as SD by RECIST, only one was classified as SD by volumetry. One of the three RECIST-based SDs had a 38.5\% reduction in baseline 3D tumor volume and was classified as PR by volumetry. One of the three RECIST-based SDs was scored as a PD by volumetry ( $26.6 \%$ increase from baseline).

Response rate Volumetric and RECIST-based data of imaging response for each patient are presented in Figs. 1 and 2. Given the variability in size of the individual lesions, each measurement is presented as a percentage of the pretreatment lesion curves for each patient with SCLC (blue curves) and NSCLC (orange). The group average for the first 4 months following treatment is shown in 1(B) and 2 (B). At 4 months post-therapy, 3D volume had decreased by 94.4\% (Fig. 1a). In contrast, the RECIST-based 2D lesion size had decreased by only by $60 \%$ during the same time period.

The average tumor 3D volume regression for the SCLC and NSCLC groups are shown in Figs. 1c and 2c. A rapid volumetric response was observed in all patients with SCLC. This rapid response is illustrated by the serial 
Fig. 1 Volumetric response to SBRT. a Percent change of 3D volume of the adrenal lesion from pre-therapy baseline over time for all patients. Curves for different patient populations are shown as follows: small cell lung cancer (SCLC) blue, nonsmall cell lung cancer (NSCLC) orange. Response curves for several NSCLC lesions are directly overlapping. Note the rapid and fairly uniform decrease in 3D volume in patients with SCLC. b Average of all volume regression curves in (a) for the first 4 months following treatment completion. c Average volumetric response for patients with SCLC and NSCLC. Patients with SCLC had a more rapid response onset. By 3 months, both groups had dramatic reductions in lesion size
Volumetric Response Following SBRT

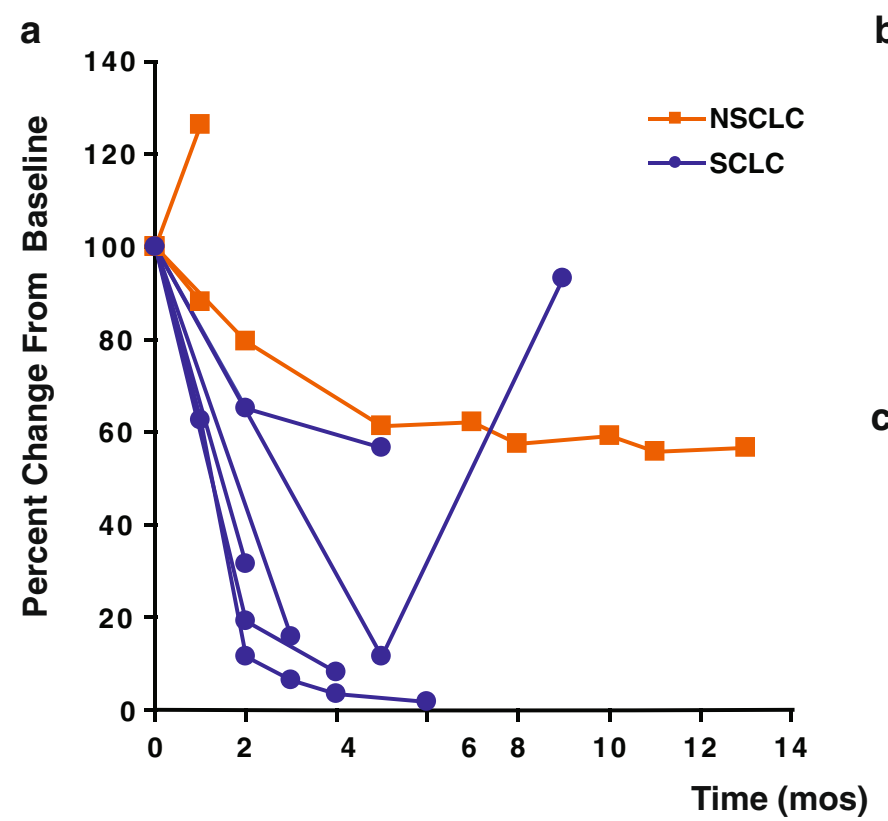

b

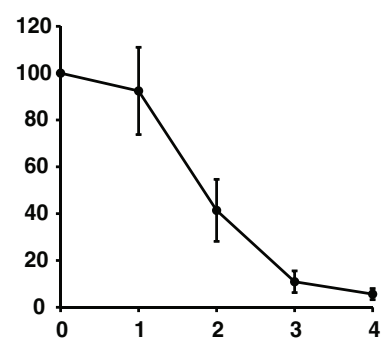

C

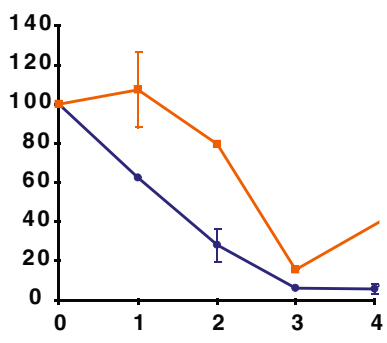

diagnostic scans in a patient with a bulky $\left(591 \mathrm{~cm}^{3}\right)$ left adrenal metastasis that showed a $38 \%$ reduction of the $3 \mathrm{D}$ tumor volume prior to the third fraction and $88.5 \%$ reduction prior to the fifth and final fraction, as shown in Fig. 3. A similar decrease in size was noted in the $2 \mathrm{D}$ measurements
(Fig. 2c); however, the rate of change was much less than for the volumetric measurements.

The rate of initial response to SBRT for both volumetric and 2D assessment methods is presented in Table 3. The mean slope of volumetric tumor regression in the SCLC

\section{RECIST-based Response Following SBRT}

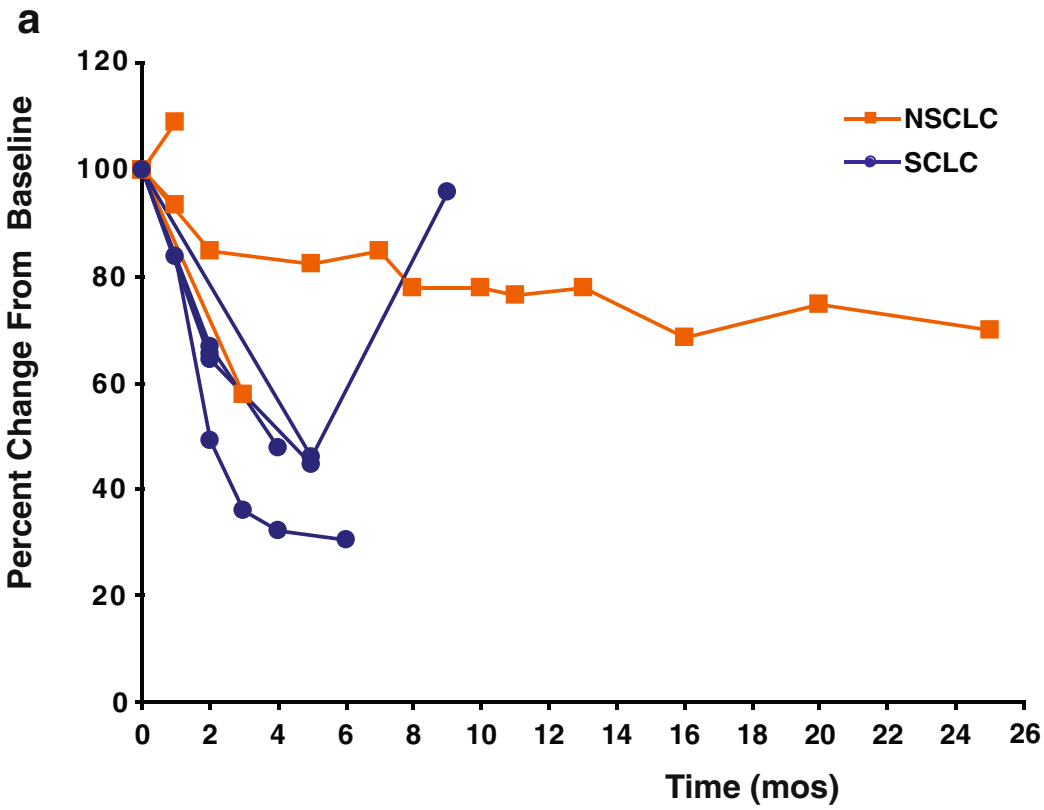

b

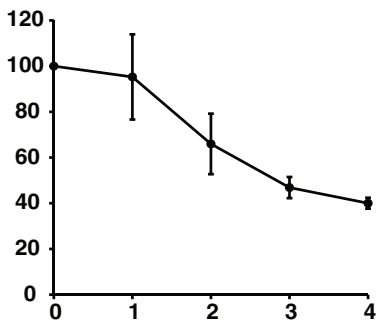

C

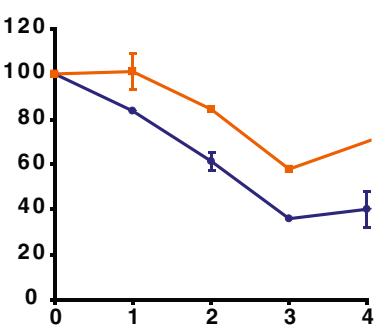

Fig. 2 Two-dimensional response to SBRT base on RECIST criteria. a Percent change of the RECIST-based 2D tumor size of the adrenal lesion from pre-therapy baseline over time for all patients. Curves for different patient populations are shown as follows: small cell lung cancer (SCLC) blue, non-small cell lung cancer (NSCLC) orange. Response curves for several NSCLC lesions are directly overlapping.
The magnitude of response is much smaller than that obtained by $3 \mathrm{D}$ volumetric measurement, shown in Fig. 1. b Average of all curves in (a) for the first 4 months following treatment completion. c Average 2D response for patients with SCLC and NSCLC. As with the individual curves, note the discrepancy in response magnitude with the $2 \mathrm{D}$ technique when comparing $\mathbf{b}$ and $\mathbf{c}$ with Fig. $1 \mathrm{~b}$ and $\mathrm{c}$ 
Fig. 3 Volumetric response in SCLC patient. This patient with a left adrenal metastasis had a protracted course of therapy. She initially received 10 Gy (5 Gy $\times 2$ fractions) prior to a break to complete a course of palliative radiotherapy for a symptomatic neck mass. Approximately 1 month later, she received an additional 10 Gy (5 Gy $\times 2$ fractions) after re-planning. Prior to her fifth and final fraction, pre-treatment conebeam CT showed poor alignment therefore planning was repeated once again to account for the significant changes in tumor volume. The dose at each time point is specified in red next to response curve. A graphical representation of her tumor with corresponding time points is shown at the bottom of the figure

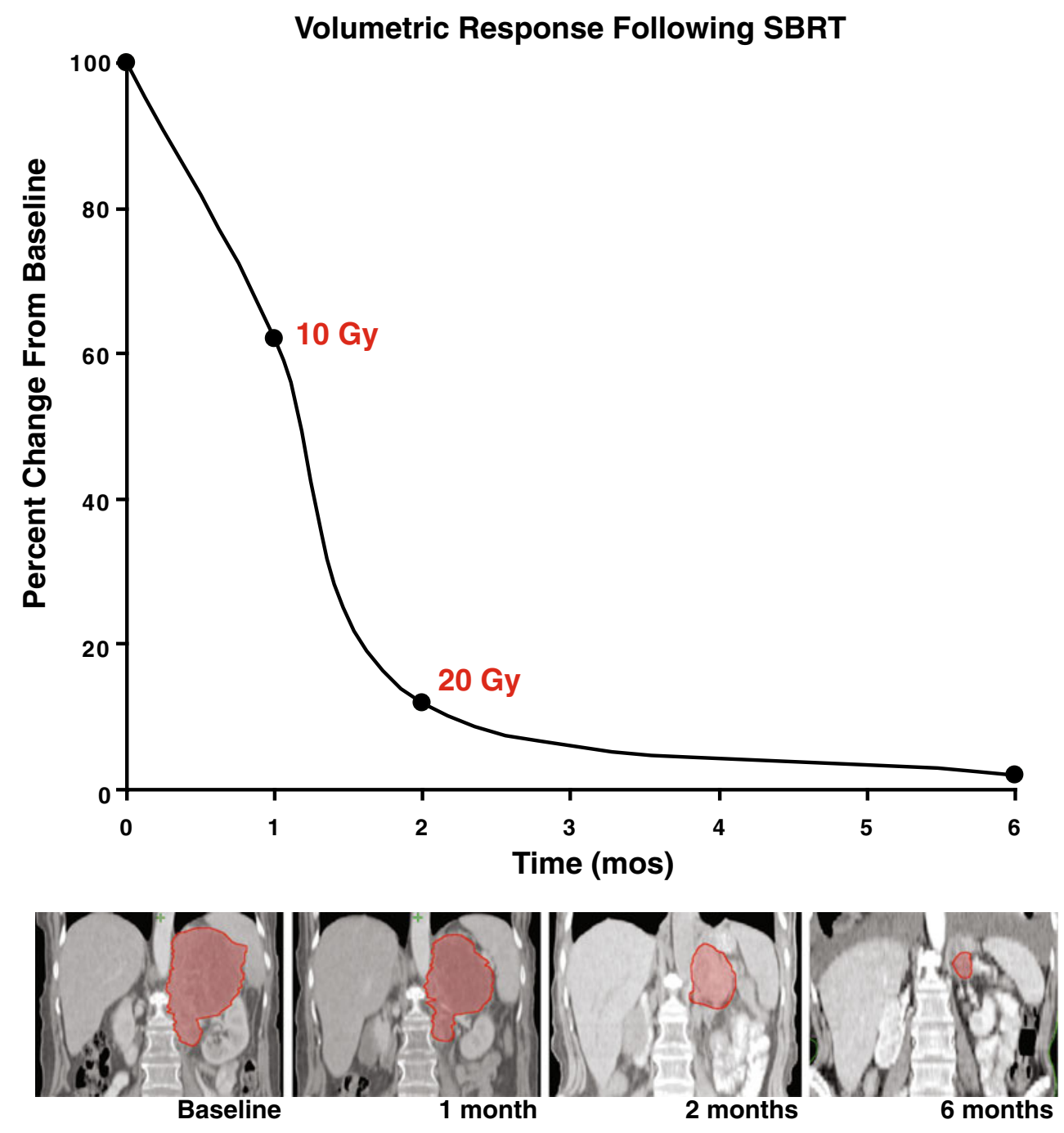

group was $-31.0 \% /$ month (range, -40.4 to -17.7 ), compared with $-5.9 \% /$ month (range, -28.1 to 26.6 ) for NSCLC patients. This difference approached but did not reach statistical significance $(p=0.06)$. The mean slope of the $2 \mathrm{D}$ tumor response in the SCLC group was $-15.8 \% /$ month

Table 3 Slope of tumor response curves for 3D volumetric vs. RECIST-based method of assessment

\begin{tabular}{lccc}
\hline Histology & \multicolumn{2}{l}{ Measurement method } & \\
\cline { 2 - 4 } & 3D volume & RECIST & $p$ value \\
\hline SCLC & -31.0 & -15.8 & 0.009 \\
NSCLC & -5.9 & -4.9 & 0.94 \\
\hline
\end{tabular}

Data reflect the slope between pre-treatment tumor measurement and first post-treatment follow-up (2-5 months). The difference between the measurement techniques was highly significant for SCLC patients ( $p=0.009)$ but not significant for NSCLC $(p=0.94)$. Abbreviations: $\mathrm{SCLC}=$ small cell lung cancer, NSCLC $=$ non-small cell lung cancer $S C L C$ small cell lung cancer, NSCLC non-small cell lung cancer (range, -17.9 to -10.8 ), compared with -4.9 (range, -14.0 to 9.1$)$ in the NSCLC group $(p=0.05)$. When the response was compared between assessment methods, the differences within the SCLC group were statistically significant $(p=0.009)$ while those in the NSCLC group were not $(p=0.94)$.

Survival The 1-year and 2-year overall post-therapy survival for all patients was $52 \%$ and $13 \%$, respectively, with a mean survival of $10.2 \pm 1.7$ months. Local control at 1 year and 2 years for all patients was 44\%. When overall survival was analyzed by histological subtype, 1-year and 2-year overall survival was $50 \%$ and $15 \%$ in the NSCLC group and $50 \%$ and $0 \%$, respectively, in the SCLC group.

Patients with metachronous adrenal metastases had a trend for longer 1-year and 2-year overall survival rates of $60 \%$ and $20 \%$ (mean survival, $10.4 \pm 2.1$ months) compared with $37.5 \%$ and $0 \%$ (mean survival, $9.8 \pm 3.1$ months) for those with synchronous metastases. This difference was not statistically significant $(p=0.77)$. 
Symptomatic response Four of the nine patients presented with symptoms of pain from their adrenal metastases. Pain relief was achieved in all symptomatic patients either during therapy or by first follow-up, and response was associated with decreased narcotic usage. Each of these patients had large lesions (mean, $332 \mathrm{~cm}^{3}$; range, 133 to $600 \mathrm{~cm}^{3}$ ) with three of the four patients demonstrating a rapid response to radiotherapy (mean percent reduction in baseline volume $70 \%$; range, $38 \%$ to $88 \%$ ). The fourth patient had bilateral lesions. On first follow-up, the larger of the two lesions $\left(600 \mathrm{~cm}^{3}\right)$ had decreased by $12 \%$ while the smaller lesion $\left(139 \mathrm{~cm}^{3}\right)$ had increased by $27 \%$. Despite this, the patient reported pain reduction while still on therapy.

Treatment toxicity No patient experienced RTOG grade $\geq 3$ acute toxicity. Two patients experienced initial nausea and vomiting while on treatment that was well controlled with oral anti-emetics. With a mean post-therapy follow-up of $7.3 \pm 7.8$ months, no grade $\geq 3$ late toxicities were observed.

\section{Discussion}

SBRT has become established as a viable non-invasive treatment option for patients with medically inoperable stage I non-small cell lung cancer [19, 25-27]. More recently, the use of SBRT has expanded to metastatic disease [28]. Initial reports on the use of SBRT in pulmonary $[10,13,16]$, liver $[9,12,14]$, and spinal $[11,22]$ metastases attests to the high local control rates and low toxicity profile for SBRT in the treatment of metastatic lesions. Such non-invasive approach with SBRT may provide a viable alternative to surgical resection of adrenal metastases, which has been generally reserved for patients with adrenal oligometastasis, controlled systemic disease, and sufficient performance status and life expectancy to tolerate surgery $[6,7]$. In our patient population of nine lung cancer patients, we have applied these principles of SBRT to treat metastatic adrenal lesions.

Response We found a favorable objective response rate of 67\% (0 CR, 6 PR via RECIST criteria) in our patients at 5 months post-therapy. This is overall consistent with the results reported for SBRT in lung and liver metastases [9, $10,12-14,16]$. Although our patient population had shorter follow-up, was entirely composed of patients with metastatic lung cancer, and had a higher rate of extensive systemic disease than other series of oligometastasis, our outcome results are consistent with the few published series of adrenal metastases $[1-5,29]$. These studies are reviewed in Table 4. A prime goal of SBRT in our population was rapid symptom relief and/or prevention of other local tumor complications. Results of symptomatic response in our patient population show that all patients had pain relief, confirming prior reports of favorable pain control rates in other published series (Table 4).

Our patients with metachronous lesions had a trend toward improved 1-year and 2-year overall survival of $60 \%$ and $20 \%$. Similar results have been published by Oshiro et al. [29] who reported greater 1-year, 2-year, and 5-year overall survival in patients with metachronous $(83 \%, 56 \%$, $56 \%)$ versus synchronous $(35 \%, 0 \%, 0 \%)$ adrenal lesions in a more selected cohort. This, in combination with our data, supports the use of SBRT in patients with metachronous adrenal metastasis from lung cancer occurring after a disease-free interval of more than 6 months.

Dose Our prescription dose (mean, 24.9 Gy; range, 20.0 $37.5 \mathrm{~Gy}$ ) and resulting $\mathrm{BED}_{10}$ (mean, 41.6; range, 28 to 65.6) was low when compared with the range of 10-60 Gy (BED 10 range, 20-151 Gy) in previous reports of SBRT for adrenal metastases (Table 4). Despite the lower dose, our subjective and objective response rates were in line with the prior studies and favorable subjective and objective responses could be achieved with our relatively lower dose than previously reported and with minimal toxicity. This result suggest that, in view of extensive critical normal structures in close vicinity, lower doses in the range of 25 to 37.5 Gy delivered in five fractions may provide effective palliation, particularly in SCLC histology.

Treatment-related toxicity Toxicity was minimal, with no grade $\geq 3$ early or late adverse effects. This finding is consistent with prior reports (Table 4) and suggests that SBRT is a promising and well-tolerated palliative modality for symptomatic adrenal metastases.

Tumor response assessment and volumetric regression rate Imaging-based response was profoundly dependent on tumor histology. The much more rapid volumetric response in SCLC compared with NSCLC histology (regression slope -31.0 vs. $-5.9 \% /$ month, Table 3 ) is consistent with the overall radiosensitivity and commonly rapid response pattern of small cell histology. This rapid response was likely accentuated by the high-dose ablative fractionation pattern of the SBRT.

We found discrepancies between 2D and 3D volumetric assessment of response in our patients. Assessment with RECIST underestimated the PR rate and classified a substantial proportion of patients as $\mathrm{SD}$, whereas $3 \mathrm{D}$ volumetry showed PR in the majority of patients and identified a patient with $\mathrm{PD}$ that had been classified as SD with RECIST. Such discrepancy between $3 \mathrm{D}$ volume and RECIST-based measurement has been demonstrated before in other tumors [30-32]. The lesser ability of RECIST to classify response or progression is likely related to the 


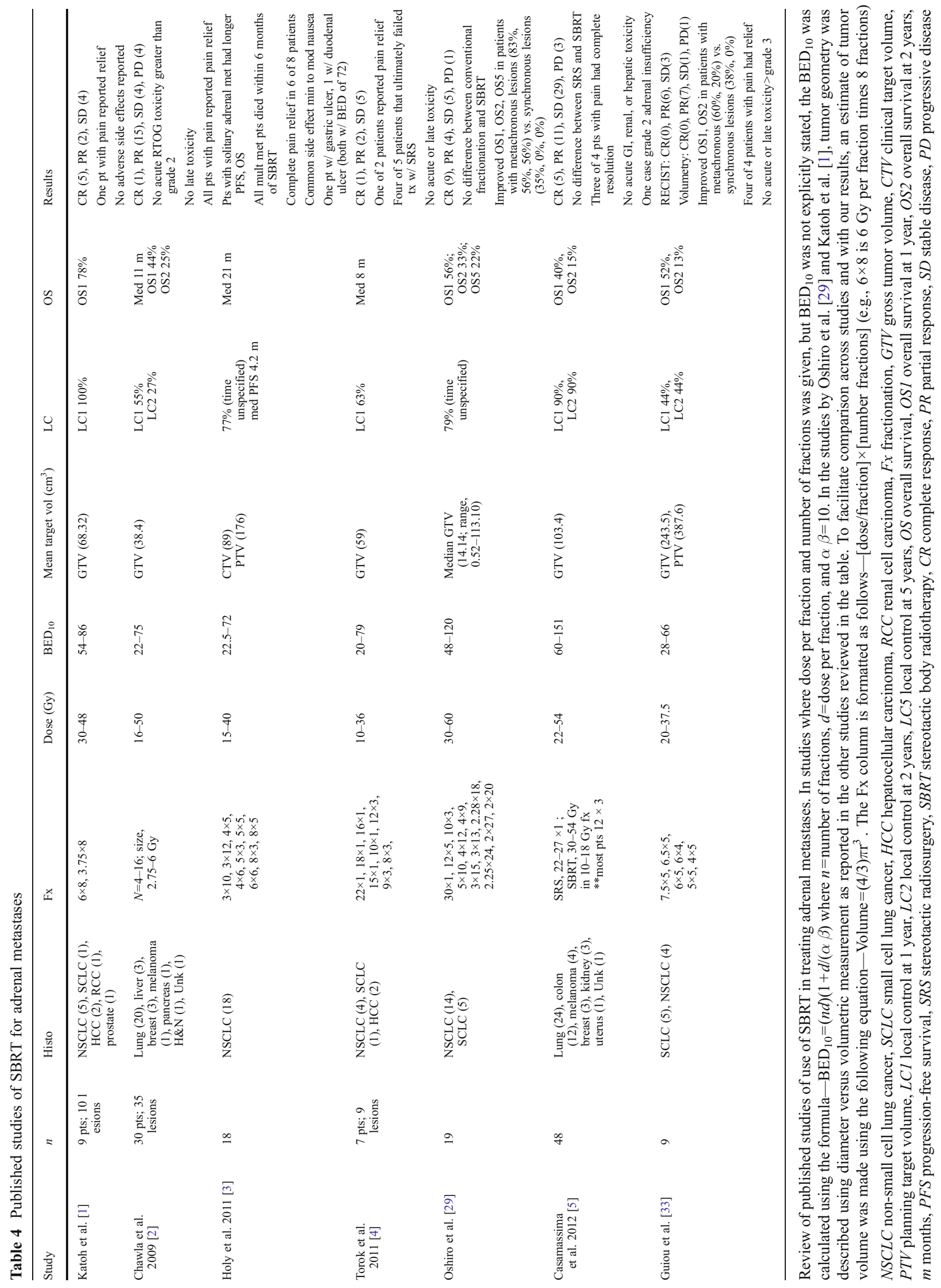


inability of two-dimensional measurements to accurately assess subtle changes in irregular tumor volumes [30]. Thus, the RECIST classification may give rise to underestimations of both tumor response rate and treatment efficacy in SBRT for adrenal lesions, as well as inadequately identify tumor progression.

Given the rapid response to SBRT seen in our SCLC patients with adrenal metastases, volumetric assessment of response during the course of SBRT delivery may be of importance. Our case of SCLC with rapid response, presented in Fig. 3, illustrates this fact. In this patient, the fast response necessitated re-planning within the course of SBRT to adapt to the rapidly regressing GTV and PTV. The adrenal gland undergoes profound displacement within the retroperitoneal space, when the tumor volume is reduced. In view of the close proximity of equally mobile critical normal structures, including kidney, stomach, and bowel, adaptive planning is of paramount importance to prevent normal tissue toxicity. Our data suggest that such volume changes and displacement of the adrenal gland can be effectively monitored with on-board CT-based imaging to enable re-planning during the treatment course.

\section{Conclusions}

SBRT is a useful, non-invasive palliative treatment option for adrenal metastases from lung cancer, providing excellent local control with minimal toxicity in a highly symptomatic and challenging complication of the disease. Volumetric tumor regression can be rapid, particularly in small cell cancer histologies, and this may influence dosimetry and the need for adaptive therapy in some patients.

\section{Conflicts of interest notification None}

\section{References}

1. Katoh N, Onimaru R, Sakuhara Y, Abo D, Shimizu S, Taguchi H, Watanabe Y, Shinohara N, Ishikawa M, Shirato H (2008) Realtime tumor-tracking radiotherapy for adrenal tumors. Radiother Oncol 87(3):418-424

2. Chawla S, Chen Y, Katz AW, Muhs AG, Philip A, Okunieff P, Milano MT (2009) Stereotactic body radiotherapy for treatment of adrenal metastases. Int J Radiat Oncol Biol Phys 75(1):71-75

3. Holy R, Piroth M, Pinkawa M, Eble MJ (2011) Stereotactic body radiation therapy (SBRT) for treatment of adrenal gland metastases from non-small cell lung cancer. Strahlenther Onkol 187(4):245-251

4. Torok J, Wegner RE, Burton SA, Heron DE (2011) Stereotactic body radiation therapy for adrenal metastases: a retrospective review of a noninvasive therapeutic strategy. Future Oncol 7 (1):145-151
5. Casamassima F, Livi L, Masciullo S, Menichelli C, Masi L, Meattini I, Bonucci I, Agresti B, Simontacchi G, Doro R (2012) Stereotactic radiotherapy for adrenal gland metastases: University of Florence experience. Int J Radiat Oncol Biol Phys 82(2):919-923

6. Raz DJ, Lanuti M, Gaissert HC, Wright CD, Mathisen DJ, Wain JC (2011) Outcomes of patients with isolated adrenal metastasis from non-small cell lung carcinoma. Ann Thorac Surg 92 (5):1788-1792, discussion 1793

7. Sancho, JJ, F Triponez, X Montet, and A Sitges-Serra (2011) Surgical management of adrenal metastases. Langenbecks Arch Surg 397:179-194

8. Paul CA, Virgo KS, Wade TP, Audisio RA, Johnson FE (2000) Adrenalectomy for isolated adrenal metastases from non-adrenal cancer. Int J Oncol 17(1):181-187

9. Herfarth KK, Debus J, Lohr F, Bahner ML, Rhein B, Fritz P, Hoss A, Schlegel W, Wannenmacher MF (2001) Stereotactic single-dose radiation therapy of liver tumors: results of a phase I/II trial. J Clin Oncol 19(1):164-170

10. Wulf J, Haedinger U, Oppitz U, Thiele W, Mueller G, Flentje M (2004) Stereotactic radiotherapy for primary lung cancer and pulmonary metastases: a noninvasive treatment approach in medically inoperable patients. Int J Radiat Oncol Biol Phys 60(1):186-196

11. Gerszten PC, Burton SA, Belani CP, Ramalingam S, Friedland DM, Ozhasoglu C, Quinn AE, McCue KJ, Welch WC (2006) Radiosurgery for the treatment of spinal lung metastases. Cancer 107(11):2653-2661

12. Mendez Romero A, Wunderink W, Hussain SM, De Pooter JA, Heijmen BJ, Nowak PC, Nuyttens JJ, Brandwijk RP, Verhoef C, Ijzermans JN, Levendag PC (2006) Stereotactic body radiation therapy for primary and metastatic liver tumors: a single institution phase I-II study. Acta Oncol 45(7):831-837

13. Okunieff P, Petersen AL, Philip A, Milano MT, Katz AW, Boros L, Schell MC (2006) Stereotactic body radiation therapy (SBRT) for lung metastases. Acta Oncol 45(7):808-817

14. Wulf J, Guckenberger M, Haedinger U, Oppitz U, Mueller G, Baier K, Flentje M (2006) Stereotactic radiotherapy of primary liver cancer and hepatic metastases. Acta Oncol 45(7):838-847

15. Gerszten PC, Burton SA, Ozhasoglu C, Welch WC (2007) Radiosurgery for spinal metastases: clinical experience in 500 cases from a single institution. Spine (Phila Pa 1976) 32(2):193-199

16. Hof H, Hoess A, Oetzel D, Debus J, Herfarth K (2007) Stereotactic single-dose radiotherapy of lung metastases. Strahlenther Onkol 183(12):673-678

17. Vautravers-Dewas C, Dewas S, Bonodeau F, Adenis A, Lacornerie T, Penel N, Lartigau E, Mirabel X (2011) Image-guided robotic stereotactic body radiation therapy for liver metastases: is there a dose response relationship? Int J Radiat Oncol Biol Phys 81(3): e39-e47

18. Lo SS, Teh BS, Mayr NA, Olencki TE, Wang JZ, Grecula JC, Lu JJ, Timmerman RD (2010) Stereotactic body radiation therapy for oligometastases. Discov Med 10(52):247-254

19. Timmerman R, Paulus R, Galvin J, Michalski J, Straube W, Bradley J, Fakiris A, Bezjak A, Videtic G, Johnstone D, Fowler J, Gore E, Choy H (2010) Stereotactic body radiation therapy for inoperable early stage lung cancer. JAMA 303(11):1070-1076

20. Zamboglou C, Messmer MB, Becker G, Momm F (2012) Stereotactic radiotherapy in the liver hilum: basis for future studies. Strahlenther Onkol 188(1):35-41

21. Rule W, Timmerman R, Tong L, Abdulrahman R, Meyer J, Boike T, Schwarz RE, Weatherall P, Chinsoo Cho L (2011) Phase I doseescalation study of stereotactic body radiotherapy in patients with hepatic metastases. Ann Surg Oncol 18(4):1081-1087

22. Ryu S, Jin R, Jin JY, Chen Q, Rock J, Anderson J, Movsas B (2008) Pain control by image-guided radiosurgery for solitary spinal metastasis. J Pain Symptom Manag 35(3):292-298 
23. Tanvetyanon T, Robinson LA, Schell MJ, Strong VE, Kapoor R, Coit DG, Bepler G (2008) Outcomes of adrenalectomy for isolated synchronous versus metachronous adrenal metastases in nonsmall-cell lung cancer: a systematic review and pooled analysis. J Clin Oncol 26(7):1142-1147

24. Eisenhauer EA, Therasse P, Bogaerts J, Schwartz LH, Sargent D, Ford R, Dancey J, Arbuck S, Gwyther S, Mooney M, Rubinstein L, Shankar L, Dodd L, Kaplan R, Lacombe D, Verweij J (2009) New response evaluation criteria in solid tumours: revised RECIST guideline (version 1.1). Eur J Cancer 45(2):228-247

25. Fakiris AJ, McGarry RC, Yiannoutsos CT, Papiez L, Williams M, Henderson MA, Timmerman R (2009) Stereotactic body radiation therapy for early-stage non-small-cell lung carcinoma: four-year results of a prospective phase II study. Int J Radiat Oncol Biol Phys 75(3):677-682

26. Onishi H, Araki T, Shirato H, Nagata Y, Hiraoka M, Gomi K, Yamashita T, Niibe Y, Karasawa K, Hayakawa K, Takai Y, Kimura T, Hirokawa Y, Takeda A, Ouchi A, Hareyama M, Kokubo M, Hara R, Itami J, Yamada K (2004) Stereotactic hypofractionated high-dose irradiation for stage I nonsmall cell lung carcinoma: clinical outcomes in 245 subjects in a Japanese multiinstitutional study. Cancer 101(7):1623-1631

27. Onishi H, Shirato H, Nagata Y, Hiraoka M, Fujino M, Gomi K, Niibe Y, Karasawa K, Hayakawa K, Takai Y, Kimura T, Takeda A, Ouchi A, Hareyama M, Kokubo M, Hara R, Itami J, Yamada K, Araki T (2007) Hypofractionated stereotactic radiotherapy (HypoFXSRT) for stage I non-small cell lung cancer: updated results of 257 patients in a Japanese multi-institutional study. J Thorac Oncol 2(7 Suppl 3):S94-S100

28. Lo SS, Fakiris AJ, Teh BS, Cardenes HR, Henderson MA, Forquer JA, Papiez L, McGarry RC, Wang JZ, Li K, Mayr NA, Timmerman RD (2009) Stereotactic body radiation therapy for oligometastases. Expert Rev Anticancer Ther 9(5):621-635

29. Oshiro Y, Takeda Y, Hirano S, Ito H, Aruga T (2011) Role of radiotherapy for local control of asymptomatic adrenal metastasis from lung cancer. Am J Clin Oncol 34(3):249-253

30. Mayr NA, Yuh WT, Taoka T, Wang JZ, Wu DH, Montebello JF, Meeks SL, Paulino AC, Magnotta VA, Adli M, Sorosky JI, Knopp MV, Buatti JM (2006) Serial therapy-induced changes in tumor shape in cervical cancer and their impact on assessing tumor volume and treatment response. AJR Am J Roentgenol 187 (1):65-72

31. Force J, Rajan A, Dombi E, Steinberg SM, Giaccone G (2011) Assessment of objective responses using volumetric evaluation in advanced thymic malignancies and metastatic non-small cell lung cancer. J Thorac Oncol 6(7):1267-1273

32. Mantatzis M, Kakolyris S, Amarantidis K, Karayiannakis A, Prassopoulos P (2009) Treatment response classification of liver metastatic disease evaluated on imaging. Are RECIST unidimensional measurements accurate? Eur Radiol 19(7):1809 1816

33. Guiou M, Mayr NA, Kim EY, Williams T, Lo SS (2012) Stereotactic body radiotherapy for adrenal metastases from lung cancer. J Radiat Oncol doi:10.1007/s13566-012-0037-8 